\title{
The maximum number of perfect matchings in graphs with a given degree sequence
}

\author{
Noga Alon* Shmuel Friedland ${ }^{\dagger \ddagger}$
}

Submitted: Mar 19, 2008; Accepted: Apr 13, 2008; Published: Apr 24, 2008

\begin{abstract}
We show that the number of perfect matchings in a simple graph $G$ with an even number of vertices and degree sequence $d_{1}, d_{2}, \ldots, d_{n}$ is at most $\prod_{i=1}^{n}\left(d_{i} !\right)^{\frac{1}{2 d_{i}}}$. This bound is sharp if and only if $G$ is a union of complete balanced bipartite graphs.
\end{abstract}

2000 Mathematics Subject Classification: 05A15, 05C70.

Keywords and phrases: Perfect matchings, permanents.

\section{Introduction}

Let $G=(V, E)$ be an undirected simple graph. For a vertex $v \in V$, let $\operatorname{deg} v$ denote its degree. Assume that $|V|$ is even, and let perfmat $G$ denote the number of perfect matchings in $G$. The main result of this short note is:

\section{Theorem 1.1}

$$
\text { perfmat } G \leq \prod_{v \in V}((\operatorname{deg} v) !)^{\frac{1}{2 \operatorname{deg} v}}
$$

where $0^{\frac{1}{0}}=0$. If $G$ has no isolated vertices then equality holds if and only if $G$ is a disjoint union of complete balanced bipartite graphs.

For bipartite graphs the above inequality follows from the Bregman-Minc Inequality for permanents of $(0,1)$ matrices, mentioned below.

The inequality (1.1) was known to Kahn and Lovász, c.f. [2, (7)], but their proof was never published, and it was recently stated and proved independently by the second author in [3]. Here we show that it is a simple consequence of the Bregman-Minc Inequality.

*School of Mathematics, Tel Aviv University, Ramat Aviv, Tel Aviv 69978, Israel, and IAS, Princeton, NJ 08540, USA, e-mail: nogaa@post.tau.ac.il. Research supported in part by the Israel Science Foundation and by a USA-Israeli BSF grant.

${ }^{\dagger}$ Department of Mathematics, Statistics, and Computer Science, University of Illinois at Chicago, Chicago, Illinois 60607-7045, USA, e-mail friedlan@uic.edu

${ }_{\ddagger}^{\ddagger}$ Visiting Professor, Fall 2007 - Winter 2008, Berlin Mathematical School, Berlin, Germany 


\section{The proof}

Let $A$ be an $n \times n(0,1)$ matrix, i.e. $A=\left[a_{i j}\right]_{i, j=1}^{n} \in\{0,1\}^{n \times n}$. Denote $r_{i}=\sum_{j=1}^{n} a_{i j}, i=$ $1, \ldots, n$. The celebrated Bregman-Minc inequality, conjectured by Minc [4] and proved by Bregman [1], states

$$
\operatorname{perm} A \leq \prod_{i=1}^{n}\left(r_{i} !\right)^{\frac{1}{r_{i}}},
$$

where equality holds (if no $r_{i}$ is zero) iff up to permutation of rows and columns $A$ is a block diagonal matrix in which each block is a square all-1 matrix.

Proof of Theorem 1.1: The square of the number of perfect matchings of $G$ counts ordered pairs of such matchings. We claim that this is the number of spanning 2-regular subgraphs $H$ of $G$ consisting of even cycles (including cycles of length 2 which are the same edge taken twice), where each such $H$ is counted $2^{s}$ times, with $s$ being the number of components (that is, cycles) of $H$ with more than 2 vertices. Indeed, every union of a pair of perfect matchings $M_{1}, M_{2}$ is a 2-regular spanning subgraph $H$ as above, and for every cycle of length exceeding 2 in $H$ there are two ways to decide which edges came from $M_{1}$ and which from $M_{2}$.

The permanent of the adjacency matrix $A$ of $G$ also counts the number of spanning 2-regular subgraphs $H^{\prime}$ of $G$, where now we allow odd cycles and cycles of length 2 as well. Here, too, each such $H^{\prime}$ is counted $2^{s}$ times, where $s$ is the number of cycles of $H^{\prime}$ with more than 2 vertices, (as there are 2 ways to orient each such cycle as a directed cycle and get a contribution to the permanent). Thus the square of the number of perfect matchings is at most the permanent of the adjacency matrix, and the desired inequality follows from Bregman-Minc by taking the square root of (2.1), where the numbers $r_{i}$ are the degrees of the vertices of $G$.

It is clear that if $G$ is a vertex-disjoint union of balanced complete bipartite graphs then equality holds in (1.1). Conversely, if $G$ has no isolated vertices and equality holds, then equality holds in (2.1), and no $r_{i}$ is zero. Therefore, after permuting the rows and columns of the adjacency matrix of $G$ it is a block diagonal matrix in which every block is an all-1 square matrix, and as our graph $G$ has no loops, this means that it is a union of complete balanced bipartite graphs, completing the proof.

\section{References}

[1] L.M. Bregman, Some properties of nonnegative matrices and their permanents, Soviet Math. Dokl. 14 (1973), 945-949.

[2] B. Cuckler and J. Kahn, Entropy bounds for perfect matchings and Hamiltonian cycles, to appear.

[3] S. Friedland, An upper bound for the number of perfect matchings in graphs, arXiv: 0803.0864v1, 6 March 2008.

[4] H. Minc, Upper bounds for permanents of (0,1)-matrices, Bull. Amer. Math. Soc. 69 (1963), 789-791. 